\title{
Comparative study on sedative and anaesthetic effects of Xylazine, Ketamine and Xylazine-Ketamine Combination in quails (Coturnix coturnix)
}

\author{
Uzma Farid Durrani, ${ }^{1}$ Asim Khalid Mahmood ${ }^{1}$, Afan Shahid ${ }^{1}$, \\ Zahid Iqbal $^{1}$, Muhammad Waqas ${ }^{1}$, Sadaf Imran ${ }^{2}$, \\ Imran Ahmed ${ }^{2}$, Rashid Hussain ${ }^{2}$ \\ ${ }^{1}$ Pet Centre, University of Veterinary and Animal Sciences, Lahore, Pakistan. \\ ${ }^{2}$ Department of Clinical Medicine and Surgery, Faculty of Veterinary Science, University of Veterinary and \\ Animal Sciences, Lahore, Pakistan.
}

\begin{abstract}
Individual and combined effects of xylazine, ketamine and their combination were studied on eighteen adult quails (Coturnix coturnix), equally divided into three groups i.e. A: xylazine (18mg/kg, IM), B: ketamine $(60 \mathrm{mg} / \mathrm{kg}, \mathrm{IM})$ and $C$ : xylazine-ketamine combination $(9 \mathrm{mg} / \mathrm{kg}-30 \mathrm{mg} / \mathrm{kg}$, IM). Xylazine, ketamine and xylazine-ketamine combination produced smooth induction of their effects in $9.7 \pm 2.11$ minutes, $7 \pm 1.56$ minutes and $1.9 \pm 0.53$ minutes, respectively. Group A attained light plane of sedation and group B attained stage II anaesthesia along with superficial analgesia. In group C, birds showed a deep anaesthesia accompanied by deep analgesia. Mean durations of sedation and anaesthesia were $40.8 \pm 3.99$ minutes, $44.1 \pm 12.99$ minutes and $77.8 \pm 18.62$ minutes in groups $A, B$ and $C$, respectively followed by a recovery phase with mean duration 22.3 \pm 2.66 minutes, $15.8 \pm 6.35$ minutes and $34.6 \pm 13.81$ minutes in groups $A, B$ and $C$, respectively. Birds in group $B$ exhibited a rough recovery while recovery was smooth in groups $A$ and $C$. It is concluded that the use of xylazine as sedative and xylazine-ketamine combination as anaesthetic is the most effective, safe and economic selection for quails especially under field conditions. Xylazine-ketamine combination is recommended as a good surgical anaesthesia to carry out painful manipulative procedures at the dosages used in this study. Use of ketamine alone is not recommended for quails.
\end{abstract}

Key Words: Xylazine, Ketamine, Xylazine-ketamine combination, Anaesthetics, Quails.

\section{Introduction}

Parenteral use of xylazine alone and in combination with anaesthetic agents is a safe, easy to handle and economic technique for birds during clinical practice. Xylazine is a potent, non narcotic, sedative, muscle relaxant and analgesic [1] [2]. Use of xylazine alone produces a light but smooth sedation and superficial analgesia [3]. High doses of xylazine produce deep sedation, leading to loss of consciousness and a light plane of anaesthesia [4]. Xylazine, detomidine and medetomidine are usually used in combination with ketamine [5] [6] [7] [8] . Combined use of alpha-2-adrenergic agonists with ketamine results in reduction of required doses, smooth induction, recovery and better muscle relaxation [9] [10]. Anaesthetic efficacy of detomidine and ketamine combination was studied in chicken and it was concluded that the use of detomidine-ketamine combination results in a fast and smooth onset of anaesthesia with a complete loss of all reflexes, good muscle relaxation [11]. Among dissociative agents, ketamine is the least potent anaesthetic agent which works for a very short duration [12]. Ketamine is a potent inhibitor of GABA binding in C.N.S. that induces amnesia and anaesthesia of stages I and II but not stage III. Due to these properties ketamine is rarely used alone because it is associated with poor muscle relaxation, muscle tremors, myotonic contractions, opisthotonus and rough recoveries; however, it may be administered alone but is more commonly used together with alpha-2-adrnergic drugs, diazepam or azaperone depending on the species involved [12]. Anaesthetic dose of ketamine alone at the dosage $(80 \mathrm{mg} / \mathrm{kg}, \mathrm{IM})$ elicits poor analgesia, lack of skeletal muscles relaxation followed by very rough recovery [3]. Under field conditions, especially in rural areas, due to very limited clinical facilities it is difficult to handle painful manipulative procedures. In this context availability of a safe, economic and effective anaesthesia is mandatory.

Main objectives of this clinical study were to determine the effect of xylazine and ketamine synergism on their individual dosages and to determine the safe and effective individual dosages of xylazine and ketamine (as combination) to produce surgical anaesthesia of $>20$ minutes for quails. 


\subsection{Bird selection}

\section{Materials And Methods}

Eighteen adult and healthy quails (Coturnix coturnix) of either sex (nine females and nine males) were purchased from the local market (Lahore, Pakistan). Their body weight ranged from 126-220 grams. All quails were randomly selected from the same flock and their age was between 1-3 years. Before the commencement of each trial, all birds were physically examined closely to judge their health status. Physical examination involved recording of body weight, temperature, respiration and heart rates, different body reflexes and evaluation of overall health status. Out of total eighteen quails; randomly selected three male and three female quails were placed in three separate cages with six birds/cage (cage size: $34 \times 30 \times 34$ inches). Cage environment was maintained as clean and stress free at a temperature $25^{\circ} \mathrm{C}-27^{\circ} \mathrm{C}$. All the quails were maintained on the same nutritional regimen allowing them ad-libitum access to feed and water.

\subsection{Experimental design}

Water and food of all quails were withheld 30 minutes prior to drug administration to eliminate possibility of emesis. This study was conducted on 18 quails that were randomly allocated to three groups titled A, B and C, each with 6 quails. Group A was treated with xylazine $(18 \mathrm{mg} / \mathrm{kg})$, group B was treated with ketamine $(60 \mathrm{mg} / \mathrm{kg})$ and group $C$ was treated with xylazine-ketamine combination $(9 \mathrm{mg} / \mathrm{kg}-30 \mathrm{mg} / \mathrm{kg})$. All treatments were administered intramuscularly using $1 \mathrm{ml}$ hypodermal syringe. Commercial details of the drugs are 2\% Injection Xylaz ${ }^{\circledR}$ (xylazine) by Farvet and 5\% Injection Calypsol® (ketamine) by Medimpex. Induction period, duration of sedation/anaesthesia, recovery period, duration and degree of analgesia, body reflexes (righting reflex, toe pinch reflex, feather plucking reflex, palpebral reflex and table knock reflex), rectal temperature, respiration and heart rate per minute were studied as parameters of this study.

\subsection{Statistical Analysis}

Data was statistically analyzed by One Way Analysis of Variance (ANOVA) and statistical difference among different trials was determined by Least Significant Difference test (L.S.D) by using SPSS version 17. Confidence interval was selected as $95 \%$ [13].

\section{Results And Discussion}

Onset duration of different drug effects, duration of sedation/anaesthesia and recovery period in groups $\mathrm{A}, \mathrm{B}$ and $\mathrm{C}$ are presented in TABLE 1.

Xylazine has a property to produce light but smooth sedation, analgesia and muscle relaxation of varying degree [14] [15] [8] [16]. In this study following xylazine injection all birds remained active during first 5 minutes followed by onset of sedation. Xylazine produced smooth but low degree of sedation along with superficial analgesia and moderate skeletal muscle relaxation in all birds. All birds remained drowsy showing sitting or standing posture with close eyes when undisturbed. Ketamine is a dissociative anaesthetic agent that produces low quality anaesthesia with no analgesia and skeletal muscle relaxation when used alone [17] [5] [8] [3]. Same findings were practically observed in present study. After administration of ketamine all birds maintained their normal behavior during initial 2-3 minutes followed by a smooth onset of drowsiness till onset of anaesthesia, and lateral recumbence. Eyes of all birds remained close during first 10 minutes after onset of anesthesia and then opened. Xylazine and ketamine combination produced better results as anaesthetic as compared to the solo use of xylazine and ketamine [8] [18] [19]. During this study xylazine-ketamine combination treated quails developed good quality anaesthesia with deep analgesia and skeletal muscles relaxation. After administration of xylazine-ketamine combination all birds went into drowsy state within initial 15-20 seconds followed by a rapid and smooth on set of general anaesthesia accompanied by deep analgesia. During xylazine-ketamine anaesthesia polyuria, hypoventilation and hypothermia were observed in all quails that persisted till 20-30 minutes prior to recovery [20] [3] [8]. In xylazine treated birds recovery was smooth but slow. Ketamine treated birds exhibited very rough recovery due to dissociative properties of ketamine and lack of skeletal muscle relaxation causing excitement, wing flapping and attempts to attain normal posture [12] [21]. Xylazine-ketamine treated birds also exhibited a smooth and quick recovery [13] [20] [19] [16]. Statistical analysis of data revealed a significant difference among duration of onset of xylazine, ketamine and xylazineketamine combination effect in all three groups (TABLE 1). Total duration and recovery period of xylazine sedation and ketamine anaesthesia were significantly different from durantion of xylazine-ktamine anaesthesia as presented in TABLE 1 [13] [3] [19]. Only xylazine and xylazine-ketmine combination produced analgesia in groups $\mathrm{A}$ and $\mathrm{C}$ while ketamine did not produce analgesia in group B. Statistically degree and duration of analgesia in groups $\mathrm{A}$ and $\mathrm{C}$ were significantly different from each other, presented in TABLE 1 [13] [3]. 


\section{Other observations}

Various body reflexes were also evaluated to determine the degree of sedation and anaesthesia. Body reflexes included palpebral reflex, toe pinch reflex, table knock reflex, pharyngeal reflex, righting reflex and feather plucking reflex. In xylazine treated birds except feather plucking reflex all reflexes were present. In ketamine treated birds only righting reflex and palpebral reflex were present whereas in xylazine-ketamine treated birds there was a complete absence of all reflexes [5] [3].

Xylazine and xylazine-ketamine combination caused hypothermia while ketamine treated birds showed hyperthermia. Both hypothermia and hyperthermia persisted until recovery or shortly thereafter (Fig. 1) [22] [12]. Respiratory distress was noted in all three groups that began to restore with the onset of recovery phase (Fig. 2) [13] [13] [16] [8].

Xylazine and xylazine-ketamine combination elicited bradycardia in all birds that persisted till recovery while ketamine treated birds suffered tachycardia that returned to normal towards the onset of recovery as presented by Fig. 3 [3]. No other untoward effect of treatments was noticed. No mortality occurred in any group. All quails attained their normal physiological status after 24 hours.

\section{Conclusion}

It is concluded that there is a highly desirable synergism between xylazine and ketamine that leads to a good quality anaesthesia and analgesia of long duration at half of their individual dosages when xylazine and ketamine are used alone. It is recommended that xylazine-ketamine combination should be used as an injectable anaesthesia for quails especially under field conditions for good quality anaesthesia with smooth induction, recovery, accompanied by deep analgesia and muscle relaxation. Solo use of ketamine is not recommended for quails because it results in a poor anaesthesia accompanied by severe side effects for quails.

\section{References}

[1]. P Lees, Sedatives, in Veterinary Applied Pharmacology and Therapeutics, (5th Ed.), (UK: Bailliere Tindall, 1991), 337-339.

[2]. K L Marx, Therapeutic agents, in Clinical Avian Medicine (Vol I), (Florida: Palm Beach, Spix Publishing, 2006), 214-342.

[3]. U F Durrani, M. Ashraf, M. A. Khan, Comparative efficacy (sedative and anaesthetic) of xylazine, ketamine and xylazine- ketamine cocktail in pigeons (Columba livia), Turkish Journal of Veterinary and Animal Sciences, 33(5), 2009, 413-417.

[4]. R Paddleford and R.C. Harvey, Alpha-2- agonists and antagonists, Veterinary Clinical North American Small Animal Practice, 29, 1999, 737-745.

[5]. J H Samour, D.M. Jones, J.A. Knight and J.C. Howlett (1984).Comparative studies of the use of some injectable anaesthetic agents in birds, Veterinary Record, 115, 1984, 6-11.

[6]. J Christensen, R.T. Fosse, O.J. Halvorsen and I. Moril, Comparison of various anaesthetic regimens in the domestic fowl, American Journal of Veterinary Research, 48, 1987, 1649-1657.

[7]. J T Lumeij and J.W. Deenik, Medetomidine-ketamine and diazepam-ketamine anesthesia in racing pigeons (Columba livia domestica)- A comparative study, Journal of Avian Medicine and Surgery,17, 2003, 191-196.

[8]. M J Gandomani, A. Tamadon, A. Mehdizadeh and H.R. Attaran, Comparison of different ketamine-xylazine combinations for prolonged anaesthesia in budgerigars (Melopsittacus undulates), Veterinary Scan, 4(1), 2009, 34.

[9]. J T Heaton and S.E. Brauth, Effects of yohimbine as a reversing agent for ketamine-xylazine anaesthesia in budgerigars, Laboratory Animal Science, 42, 1992, 54-56.

[10]. F K Mohammad, M.S. Al-Badrany and A.M. Al-Hassan, Detomidine-ketamine anaesthesia in chicken, Veterinary Record, 133, 1993, 192.

[11]. N H Booth, and L.E. McDonald, Intravenous and Other Parenteral Anaesthetics, in Veterinary pharmacology and therapeutics (6th Ed.) (USA: Iowa State University press, 1988), 253-264.

[12]. P Sandmeier, Evaluation of medetomidine for short term immobilization of domestic pigeons (Columba livia) and Amazon parrot (amazon specie), Journal of Avian Medicine and Surgery, 14, 2000, 8-14.

[13]. G W Snedecor and W.J. Cohran, Statistical Methods. In: Ames, Iowa (Iowa State University Press, 1967) 229-338.

[14]. T Kanda and Y. Hikasa, Neurohormonal and metabolic effects of medetomidine compared with xylazine in healthy cats, Canadian Journal of Veteterinary Research, 72 (3), 2008, 278-286.

[15]. U F Durrani, M.A. Khan, S.S. Ahmad, Comparative efficacy (sedative and anaesthetic) of detomidine, ketamine and detomidineketamine cocktail in pigeons (Columba livia), Pakistan Veterinary Journal, 28(3), 2008, 115- 118.

[16]. S Y Kodera, M. Yoshida, K. Dezaki, T. Yada, T. Murayama, M. Kawakami, M. Kakei 2013, Inhibition of insulin secretion from rat pancreatic islets by dexmedetomidine and medetomidine, two sedatives frequently used in clinical settings, Endocrinology Journal, 60 (3), 2013, 337-346.

[17]. U F Durrani, M. Ashraf, A. Khalid, Comparative efficacy of detomidine and detomidine-ketamine cocktail in quails, Pakistan Veteterinary Journal, 25(4), 2005, 197-199.

[18]. J J De Lucas, C. Rodriguez and M. Marin, Pharmacokinetics of intramuscular ketamine in young ostriches pre-medicated with romifidine, Journal of Veterinary Medicine, 54, 2007, 48-50.

[19]. A Barsheshet, M. Garty, E. Grossman, A. Sandach, B.S. Lewis, S. Gottlieb, A. Shotan, S. Behar, A. Caspi R. Schwartz, A. Tenenbaum, J. Leor, Admission blood glucose level and mortality among hospitalized nondiabetic patients with heart failure. Archives of Internal Medicine, 166 (15), 2006, 1613-1619.

[20]. H Fattahian and H. Moosavian, H. Mohyeddin and H Akbarin, R Moridpour, Blood glucose concentration profile after single dose of dexamethasone during operation in dogs, Comparative Clinical Pathology, 21, 2012, 957-960.

[21]. U F Durrani, M. Khan, A. Khalid, Study of anaesthetic efficacy of detomidine-ketamine cocktail in budgerigars, Zoos Print Journal of India, 21(9), 2006, 2416-2417.

[22]. A M Shakoor, G. Athar and N.I. Chaudhry, Anaesthesia in avian species, Pakistan Veterinary Journal, 16, 1996, 140-143. 
Comparative study on sedative and anaesthetic effects of Xylazine, Ketamine and Xylazine-Ketamine

Table 1: Duration of Onset of Action, Duration of Effect, Duration of Analgesia and Recovery Period

\begin{tabular}{ccccc|}
\hline Groups & Onset & Duration & Recovery & Analgesia \\
A & $9.7 \pm 2.11^{\mathrm{a}}$ & $40.8 \pm 3.99^{\mathrm{a}}$ & $22.3 \pm 2.66^{\mathrm{a}}$ & $25.5 \pm 7.78^{\mathrm{a}}$ \\
B & $07 \pm 1.56^{\mathrm{b}}$ & $44.1 \pm 12.99^{\mathrm{a}}$ & $15.8 \pm 6.35^{\mathrm{a}}$ & 0 \\
C & $1.9 \pm 0.53^{\mathrm{c}}$ & $77.8 \pm 18.62^{\mathrm{c}}$ & $34.6 \pm 13.81^{\mathrm{c}}$ & $95.5 \pm 7.78^{\mathrm{c}}$
\end{tabular}

*Values with different superscripts under each parameter are significantly different from each other $(\mathrm{P}<0.05)$.

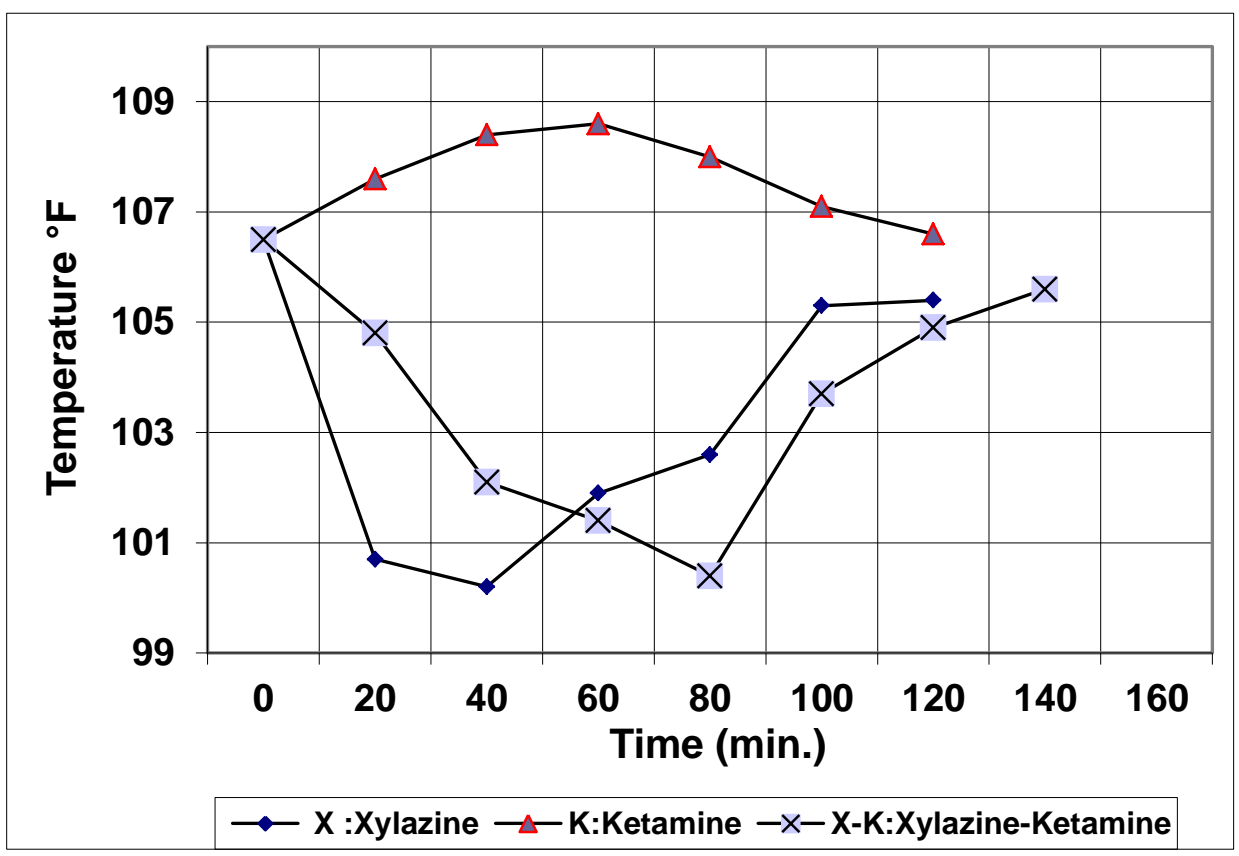

Figure 1: Comparison of Body Temperature at Various Time Intervals after Drug Administration in Groups A, B \& C

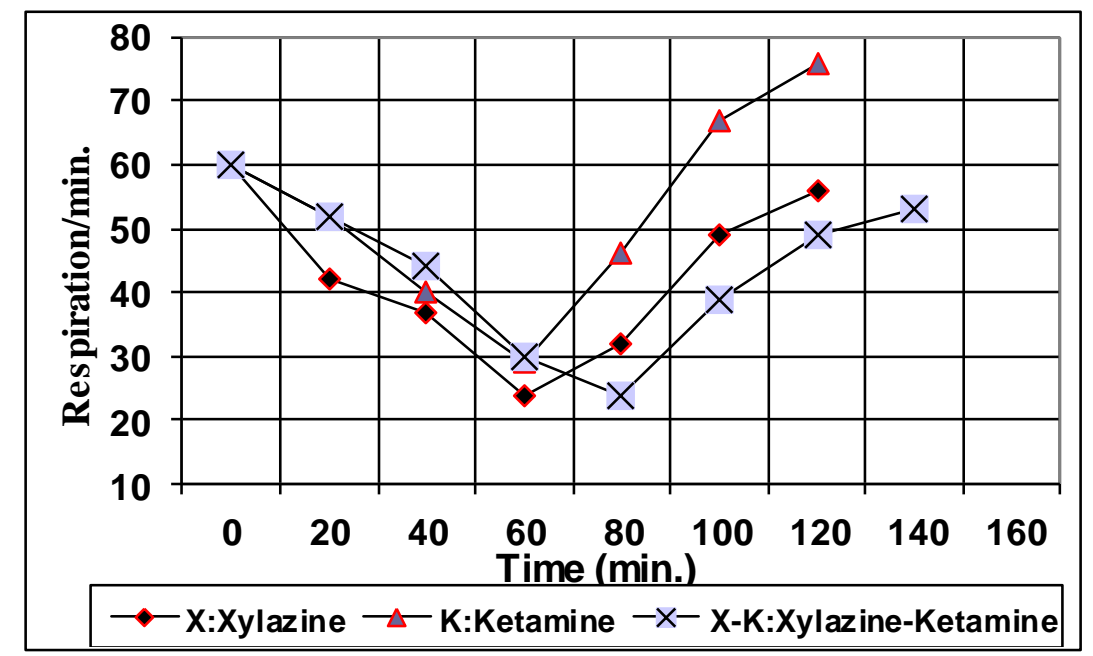

Figure 2: Comparison of Respiration Rate at Various Time Intervals after Drug Administration in Groups A, B \& C. 


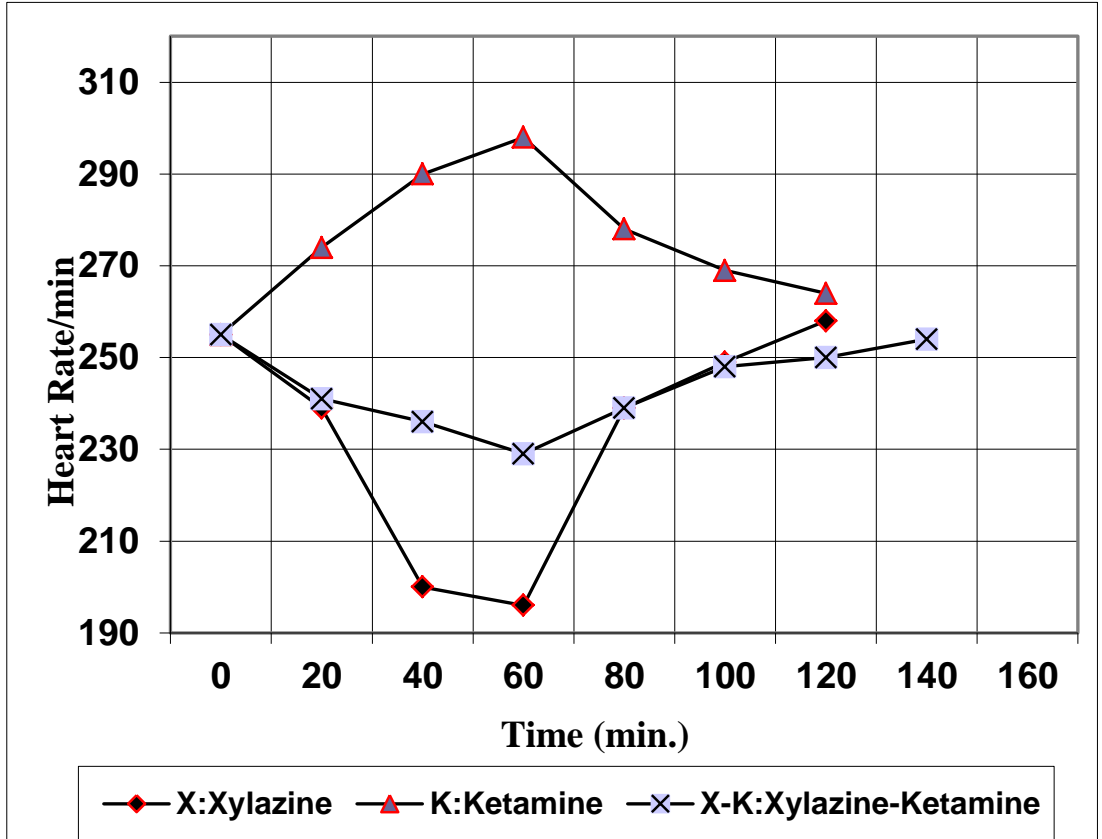

Figure 3: Comparison of Heart Rate at Various Time Intervals after Drug Administration in Groups A, B \& $\mathbf{C}$ 